\title{
Clinical application of one-position complete retroperitoneal laparoscopic radical resection of renal pelvis and ureteral carcinoma
}

\section{Yongshuang Xiao}

Xuzhou Medical College Affiliated Hospital

Wei Du

Xuzhou Medical College Affiliated Hospital

\section{Yulong Hou}

Xuzhou Medical College Affiliated Hospital

\section{Hailong Li}

Xuzhou Medical College Affiliated Hospital

\section{Shuofeng Li}

Xuzhou Medical College Affiliated Hospital

\section{Rumin Wen ( $\nabla$ wenrumin163@163.com )}

Xuzhou Medical College Affiliated Hospital

\section{Research article}

Keywords: upper tract urothelial carcinoma,UTUC, radical resection, retroperitoneal laparoscopic, oneposition

Posted Date: January 24th, 2020

DOI: https://doi.org/10.21203/rs.2.21793/v1

License: (c) (i) This work is licensed under a Creative Commons Attribution 4.0 International License. Read Full License 


\section{Abstract}

Background: To explore the surgical technique and the clinical efficacy of complete retroperitoneal laparoscopic radical resection in patients with upper tract urothelial carcinoma (UTUC).

Methods: A total of 11 patients ( 6 males and 5 females) newly diagnosed with UTUC with T1/T2 were enrolled in this study who underwent one-position complete retroperitoneal laparoscopic radical resection in our hospital from March 2016 to December 2018. Among them, 8 cases were of renal pelvis carcinoma and 3 cases were of ureteral carcinoma. The average age was 57.5 years (range, $44-78$ years). All patients were presented with painless gross hematuria. CTU and ureteroscopy were used to diagnose.

Results All the 11 patients had successful operations without open transition. The mean operating time was $85 \mathrm{~min}$ (range 60 to $115 \mathrm{~min}$ ). The mean amount of intraoperative bleeding was below $90 \mathrm{ml}$. The postoperative hospital stay was 3-7 days, with an average of 5 days. There was no obvious complication after operation. No local tumor recurrence or new bladder implantation metastasis has already been observed with the postoperative follow-up time in 8 to 40 months.

Conclusion One-position complete retroperitoneal laparoscopic radical resection of UTUC is safe and feasible. The curative effect is affirmative. It has the conspicuous advantages in less bleeding during operation, minimally invasive, no posture change, quicker recovery with little disturbance of abdominal organ and shorter postoperative hospital stay, which could particularly reduce workload of operating room nurses, without any extra postoperative complications.

\section{Background}

Upper tract urothelial carcinoma (UTUC) is a relatively rare urological malignant tumor, accounting for $5 \%-6 \%$ of upper urinary tract tumors [1]. In recent years, retroperitoneal laparoscopy nephroureterectomy has been used progressively as a minimally invasive treatment substituted for open surgery in China [2], the standard resection limitation of which generally includes the total nephroureterectomy with excision of bladder cuff [3]. However, prevalently, patient's posture should be changed from side-lying position to horizontal position during this surgery after kidney and upper ureter dissociated, in order for the resection of distal ureter and bladder near ureteral orifice. Although transperitoneal approach could also accomplish this laparoscopic procedure without posture changes in western countries, pneumoperitoneum interferences on abdominal organs reflecting in abdominal pain, abdominal distension, bacterial translocation and abdominal cavity implantation metastasis has been paid more great attentions in recent study. In this study, we attempt to suggest the complete retroperitoneal laparoscopic radical resection in patients with UTUC. On the basis of mature retroperitoneal laparoscopy procedures, researching domestic experience and lessons, from March 2016 to December 2018, 11 patients in our hospital treated for UTUC underwent complete retroperitoneal laparoscopic radical resection. It brings satisfactory outcomes in terms of short operating time, minimal tissue dissections, less bleeding, rare postoperative gastrointestinal symptoms, early catheter removal, without the increase 
of tumor recurrence and metastasis. The corresponding procedure and results has been summarized as follows.

\section{Methods}

\subsection{General data}

This group is of 11 patients, consisting of 6 male patients and 5 female patients, with a mean age of 57.5 years (range, 44-78 years). Among them, 8 cases had renal pelvic carcinoma, 3 cases had primary ureter carcinoma (7 of them had right-sided lesions, and the remaining 4 had left-sided). The irritative symptoms of all patients were gross hematuria with or without affected side waist abdomen ache. CTU and intraoperative ureteroscopy were used to diagnose with the staging of clinical pathology from cT1 to cT2. All patients did not receive chemotherapy, radiotherapy or biological immunotherapy before surgery, and there was no serious underlying disease and history of upper urinary tract surgery. Informed consent was signed for all patients and the study was approved by the Medical Ethics Committee of our hospital.

\subsection{Operative technique}

All patients received the operation under general anesthesia. The patients were placed on the lateral side. The waist bridge was heightened adequately so as to ensure the patient's waist fully extended. A 10-12 $\mathrm{mm}$ skin incision was made at the intersection of the vertical line perpendicular to the anterior superior iliac spine to the dorsal side and the lower edge of the costal margin to indicate blunt dissection of the lumbar fascia, and the peritoneal dilator was fully expanded and the retroperitoneal space was established to insert a $12 \mathrm{~mm}$ sleeve. A $10 \mathrm{~mm}$ skin incision was made at the intersection of the horizontal and the anterior line of the anterior superior iliac spine, and a $10 \mathrm{~mm}$ cannula was used to place the lens. The pneumoperitoneum was established and the pressure was $12-15 \mathrm{mmHg}(1 \mathrm{mmHg}=0.133 \mathrm{kPa})$. The third and fourth cannulas were laparoscopically placed, forming a parallelogram with the first two cannulas (Figure 1). The extraperitoneal fat was removed using an ultrasonic scalpel. The boundary between the peritoneal reflex and the perirenal fascia was exposed; the anatomical landmarks such as the psoas muscle, the perirenal fascia and the peritoneal regurgitation were identified. The perirenal fascia was dissected from the dorsal side of the kidney with an ultrasonic knife and separated. The lateral fascia was cut along the anterior edge of the psoas muscle into the anterior space of the psoas muscle and the lumbar muscle; the dorsal side of the perirenal fascia was isolated. The ureter was found along the surface of the psoas muscle and clamped with Hem-o-lok in the upper ureter (Figure 2). From the level of the diaphragm, down to the level of the iliac vessels, efforts were made to try to push the kidney to the ventral side. The renal hilum was bluntly separated from the posterior kidney by an aspirator, and the renal artery was revealed and separated, and the cutting was made with Hem-o-lok. Efforts continued to detach from the distal end of the ureter. The lens was reinserted from a $12 \mathrm{~mm}$ cannula towards the pelvic cavity, and the ureter was continued to the inner segment of the bladder wall with an ultrasonic knife, and part of the bladder wall muscle layer was cut to reveal a large portion of the swelling. The ureter and part of the bladder wall were lifted, multiple Hem-o-lok clips were used for 
bladder sleeve resection, and the incision in the bladder was sutured with absorbable sutures (Figure 3). The intact gross specimen of the renal ureter (Figure 4) was removed from the $12 \mathrm{~mm}$ cannula under the enlarged costal margin and the end of the ureter and the cystic resection of the bladder were examined for completeness. After the bleeding was stopped and the retroperitoneal drainage tube was placed, the incision was sutured.

\section{Results}

Complete retroperitoneal laparoscopic radical resection in all these patients was carrying out successfully without converting to open surgery. The mean surgery time was $85 \mathrm{~min}$ (range 60 to $115 \mathrm{~min}$ ). The mean amount of intraoperative bleeding was below $90 \mathrm{ml}$. The postoperative hospital stay was 3-7 days, with an average of 5 days. There were no obvious complications afterwards. No local tumor recurrence or new bladder implantation metastasis has already been observed with the postoperative follow-up time in 8 to 40 months.

\section{Discussion}

UTUC accounts for about $5 \%$ of urothelial carcinoma [4], with the characteristics of high recurrence and multi-center occurrence. With the advancement of laparoscopic techniques and the accumulation of operative experience, laparoscopic total nephroureterectomy with excision of bladder cuff have been applicable to a growing number of pelvic ureteral cancer patients in many centers since it was successfully performed by Clayman et al in 1991[5, 6]. Recently, laparoscopic nephroureterectomy has replaced open surgery as standard surgical treatment for upper urinary tract epithelial carcinoma bacause of the less intraoperative bleeding, minimal invasion, quicker recovery and shorter postoperative hospital stay[7-11].

At present there are many surgical approaches for laparoscopic nephroureterectomy. The most common method was the Bishoff method[12], that is, laparoscopic renal and upper ureter resection in the upper abdomen, and lower ureter and partial bladder incision in the lower abdomen. But this procedure is not completely done under the laparoscopy. In 1999, Gill et al $[13,14]$ reported a complicated laparoscopic radical resection of renal pelvic ureteral cancer. The ureteral bladder wall was firstly treated with lithotomy position, and then supine position was performed through the renal and abdominal ureteral resection surgery. In this operation, two $5 \mathrm{~mm}$ cannulas were inserted into the bladder by puncturing the lower abdomen into the bladder, and another surgeon completed ureteral intubation by transurethral insertion of cystoscope. Under the help of grasping forceps of bladder, the inner segment of ureteral bladder wall was cut about $3-4 \mathrm{~cm}$. Then the lasso was placed at the distal end of the free ureteral wall and tightened. Then the electrotomy was continued to remove the entire ureteral wall from the bladder. Ligation of the lasso effectively prevents the leakage of urine in the upper urinary tract, but it could not prevent the leakage of urine containing tumor cells in the bladder, so it still remains the risk of tumor spread. In response to the problem of leaking urine in the bladder and ureteral stump, McDougall et al.[15] proposed a solution: using a linear cutting occluder to perform a sleeve-like resection of the bladder 
tissue around the ureteral opening. At the same time, the stump was sealed to avoid the urine leakage and prevent the spread of tumor as possible. This report examined the effects of 10 patients and found no tumor spread after surgery. Chandhoke et al[16] followed up for 3-9 months in patients undergoing bladder-sleeve resection with a straight-lined closure. No resection of bladder staples and formation of stones were found after cystoscopy.

In china, retroperitoneal laparoscopy is broader adopted by urologist due to minimal invasion and minimum interference with abdominal organs compared to transperitoneal laparoscopy. By way of exception the retroperitoneal laparoscopic for pelvic ureteral carcinoma, after the resection of renal and ureteral by laparoscopy in position on uninjured side, it should be moved to the supine position in order for the further excision of bladder cuff, which indeed is a time-consuming process and may be a waste of both medical and human resources.

Accordingly, in this study, based on the traditional retroperitoneal laparoscopic radical nephrectomy, a modified four-hole method complete retroperitoneal laparoscopic radical nephrectomy for pelvic and ureteral cancer was performed. After radical nephrectomy and upper ureter dissection, we added the forth Trocar in the ventral of the sight Trocar which had been located over the iliac crest at the same level. Then, the lens was relocated from the dorsal subcostal Trocar towards the pelvic cavity, and the distal ureter was continued dissociating downward to the bladder wall. Afterwards, multiple Hem-o-lok clips were used for the bladder sleeve resection and absorbable titanium clips were used for clipping the distal bladder incision.

At present, there rarely have reports of the application about complete retroperitoneal laparoscopic radical resection of renal pelvis and ureteral carcinomar. The technique we introduced has the following advantages: 1) Through the establishment of retroperitoneal approach, the anatomical landmark is easy to identify with clear visual field; 2) The application of absorbable clips of the bladder incision reduces the possibility of postoperative bladder calculus; 3) Four-hole operation is beneficial to adjust the operating field to the best; 4) The entire surgical procedure without the need of posture changes, shortening the time of operation and reducing potential safety risks during the movement of patients; 5) On the basis of the retroperitoneal laparoscopic nephrectomy, no additional surgical instrument or special equipment should be added so that there has not been a rise in cost.

Through this study, we have some enlightenments as follows: 1) After finding the upper part of the ureter, Hem-o-lok should be used to clamp the distal ureter in order for blocking the implantation metastasis caused by the influx of proximal urine containing cancer cells; 2) Specimen bag should be used for removing the excision to decrease the occurrence of implantation metastasis to abdominal incision after complete resection; 3) Operation area should be washed repeatedly with warm sterilized water at about $42^{\circ} \mathrm{C}$ to destroy the potential escaped cancer cells; 4) Although we do not recommend this new surgical technique in lower ureteral cancer because of the relatively difficult exposure of distal ureter, we are trying to resect the lower ureter and partial bladder first before the dissociate of kidney and upper ureter. The feasibility and the effect on prognosis are still in observation. 


\section{Conclusions}

In summary, completing retroperitoneal laparoscopic nephroureterectomy for the treatment of UTUC has its advantage in no posture change, less bleeding during operation, minimally invasive, quicker recovery and shorter postoperative hospital stay without any postoperative complications such as bladder calculi, higher local recurrence and vesical implantation metastasis probability of cancer.

As the limited number of specimen available and insignificant follow-up time, further follow-up observation is required to confirm the long-term efficacy of the treatment.

\section{Declarations}

\section{Funding}

None

\section{Availability of data and materials}

Not applicable.

\section{Ethics Approval and Consent to Participate}

Affiliated Hospital of Xuzhou Medical University ethics committee. The study had the consent of the participants and was documented in a medical document with surgical informed consent.

\section{Consent for publication}

Not applicable.

\section{Conflict of interest}

The authors declare that they have no conflict of interest.

\section{Acknowledgements}

Declared none.

\section{Authors Contributions}

Yongshuang Xiao analyzed and explained the patient data about the operation method, the main performer of the operation; Yulong Hou and Wei Du performed data collection and manuscript writing. Li Hailong participated in the translation and guidance of the article; Shuofeng Li and Rumin Wen participated in the design of the experiment and directed the writing. The final draft read and endorsed by all authors. 


\section{Authors' Information}

Yulong Hou and Wei Du are both graduate students in urology of Xuzhou medical university. Yongshuang Xiao and Hailong $\mathrm{Li}$ are deputy chief physicians and graduate supervisors of the Affiliated Hospital of Xuzhou Medical University. Rumin Wen is a professor and graduate tutor of the Affiliated Hospital of Xuzhou Medical University.

\section{Abbreviations}

UTUC

upper tract urothelial carcinoma

CTU

computed tomography urography

\section{References}

1.

Kang HW, Jung HD, Ha YS, Kim TH, Kwon TG, Byun SS, Yun SJ, Kim WJ, Choi YD. Preoperative Underweight Patients with Upper Tract Urothelial Carcinoma Survive Less after Radical Nephroureterectomy. J Korean Med Sci. 2015;30(10):1483-9. doi:10.3346/jkms.2015.30.10.1483. 2.

Matsumoto K, Hirayama T, Kobayashi K, Hirano S, Nishi M, Ishii D, Tabata K, Fujita T, Iwamura M. Laparoscopic Retroperitoneal Nephroureterectomy is a Safe and Adherent Modality for Obese Patients with Upper Urinary Tract Urothelial Carcinoma. Asian Pac J Cancer Prev. 2015;16(8):3223-7. doi:10.7314/apjcp.2015.16.8.3223.

3.

Mandalapu RS, Matin SF. Contemporary Evaluation and Management of Upper Tract Urothelial Cancer. Urology. 2016;94:17-23. doi:10.1016/j.urology.2015.12.035.

4.

Verges DP, Lallas CD, Hubosky SG, Bagley DH Jr. Endoscopic Treatment of Upper Tract Urothelial Carcinoma. Curr Urol Rep. 2017;18(4):31. doi:10.1007/s11934-017-0675-x. 5 .

Roupret M, Babjuk M, Comperat E, Zigeuner R, Sylvester RJ, Burger M, Cowan NC, Bohle A, Van Rhijn BW, Kaasinen E, Palou J, Shariat SF. European Association of Urology Guidelines on Upper Urinary Tract Urothelial Cell Carcinoma: 2015 Update. Eur Urol. 2015;68(5):868-79. doi:10.1016/j.eururo.2015.06.044. 6.

Clayman RV, Kavoussi LR, Figenshau RS, Chandhoke PS, Albala DM. Laparoscopic nephroureterectomy: initial clinical case report. J Laparoendosc Surg. 1991;1(6):343-9. doi:10.1089/Ips.1991.1.343.

7.

Liu JY, Dai YB, Zhou FJ, Long Z, Li YH, Xie D, Liu B, Tang J, Tan J, Yao K, He LY. Laparoscopic versus open nephroureterectomy to treat localized and/or locally advanced upper tract urothelial carcinoma: 
oncological outcomes from a multicenter study. BMC Surg. 2017;17(1):8. doi:10.1186/s12893-016-0202$\mathrm{X}$.

8.

Kim CH, Kim KT, Kim KH, Yoon SJ. Hand-assisted retroperitoneoscopic nephroureterectomy with bladder cuffing after preperitoneal and retroperitoneal perivesical ballooning. Korean J Urol. 2014;55(1):29-35. doi:10.4111/kju.2014.55.1.29.

9 .

Tai HC, Lai MK, Chung SD, Huang KH, Chueh SC, Yu HJ. Intermediate-term oncological outcomes of handassisted laparoscopic versus open bilateral nephroureterectomy for dialysis and kidney transplant patients with upper urinary tract urothelial carcinoma. J Endourol. 2009;23(7):1139-44. doi:10.1089/end.2008.0162.

10.

Rassweiler JJ, Schulze M, Marrero R, Frede T, Palou Redorta J, Bassi P. Laparoscopic nephroureterectomy for upper urinary tract transitional cell carcinoma: is it better than open surgery? Eur Urol. 2004;46(6):690-7. doi:10.1016/j.eururo.2004.08.006.

11.

Zou L, Zhang L, Zhang H, Jiang H, Ding Q. Comparison of post-operative intravesical recurrence and oncological outcomes after open versus laparoscopic nephroureterectomy for upper urinary tract urothelial carcinoma. World J Urol. 2014;32(2):565-70. doi:10.1007/s00345-013-1160-x. 12.

McDougall EM. (2000) Atlas of Laparoscopic Retroperitoneal Surgery. 165 (3):1057-1058. 13.

Gill IS, Soble JJ, Miller SD, Sung GT. A novel technique for management of the en bloc bladder cuff and distal ureter during laparoscopic nephroureterectomy. J Urol. 1999;161(2):430-4.

14.

Gill IS, Sung GT, Hobart MG, Savage SJ, Meraney AM, Schweizer DK, Klein EA, Novick AC. Laparoscopic radical nephroureterectomy for upper tract transitional cell carcinoma: the Cleveland Clinic experience. J Urol. 2000;164(5):1513-22.

15.

Bastiampillai R, Lavallee LT, Cnossen S, Witiuk K, Mallick R, Fergusson D, Schramm D, Morash C, Cagiannos I, Breau RH. Laparoscopic nephroureterectomy is associated with higher risk of adverse events compared to laparoscopic radical nephrectomy. Can Urol Assoc J. 2016;10(3-4):126-31. doi:10.5489/cuaj.3362.

16.

Chandhoke PS, Clayman RV, Kerbl K, Figenshau RS, McDougall EM, Kavoussi LR, Stone AM. Laparoscopic ureterectomy: initial clinical experience. J Urol. 1993;149(5):992-7. doi:10.1016/s00225347(17)36277-8.

\section{Figures}




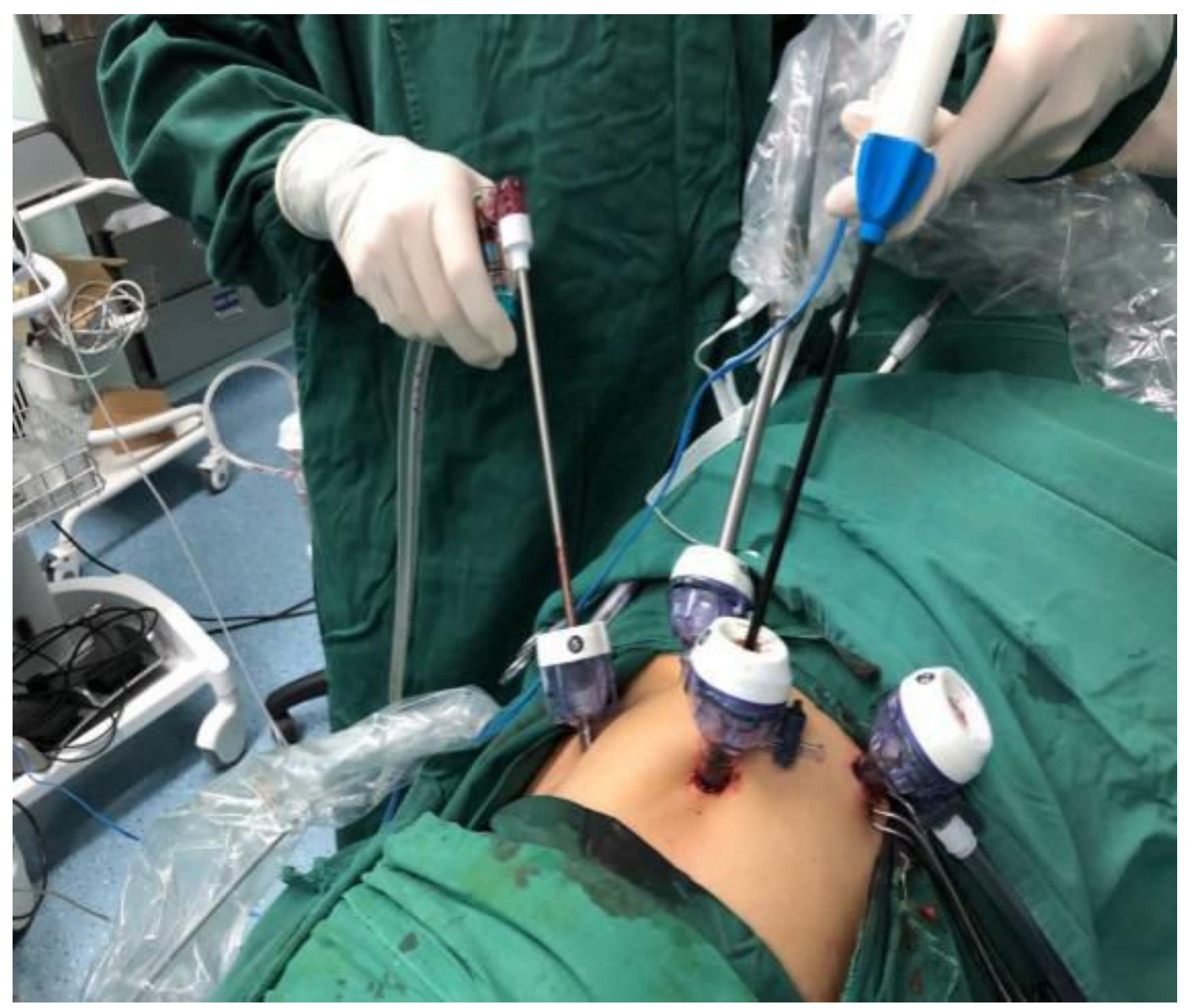

Figure 1

The third and fourth cannulas were laparoscopically placed, forming a parallelogram with the first two cannulas. 


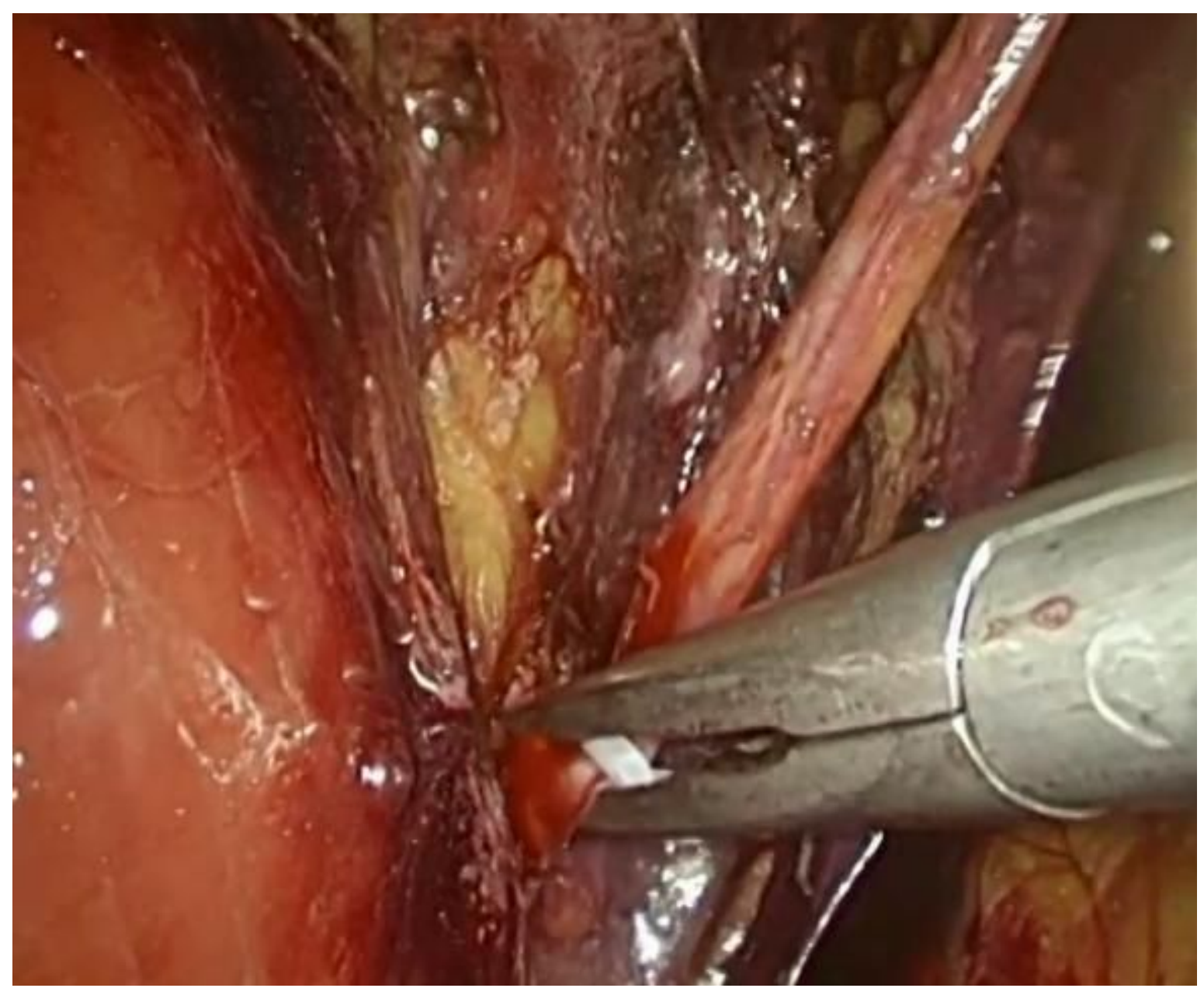

Figure 2

The ureter was found along the surface of the psoas muscle and clamped with Hem-o-lok in the upper ureter. 


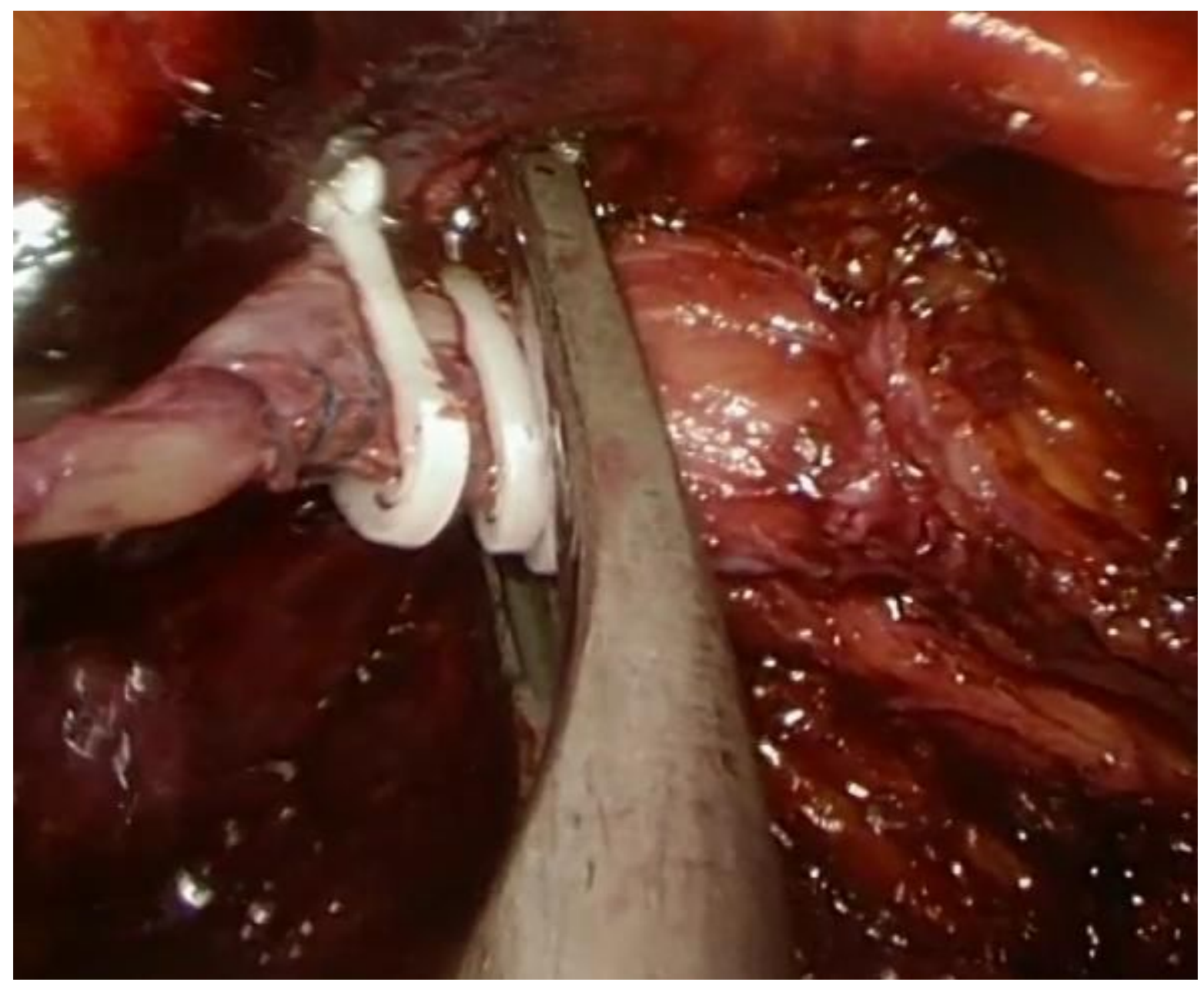

\section{Figure 3}

The ureter and part of the bladder wall were lifted, multiple Hem-o-lok clips were used for bladder sleeve resection, and the incision in the bladder was sutured with absorbable sutures. 


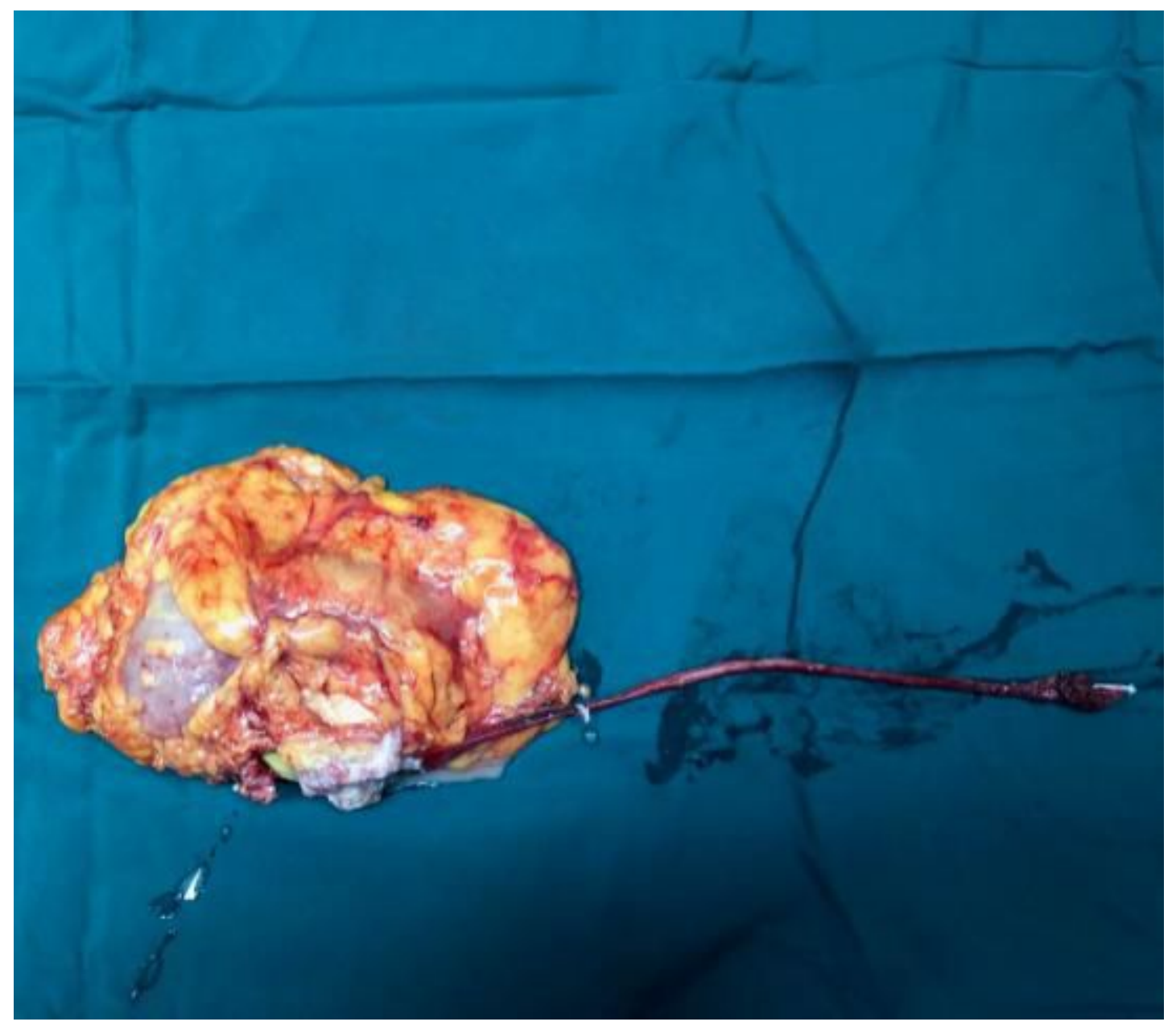

\section{Figure 4}

The intact gross specimen of the renal ureter was removed from the $12 \mathrm{~mm}$ cannula under the enlarged costal margin 